\title{
Radiation Transport Calculation of the UGXR Collimators for the Jules Horowitz Reactor (JHR)
}

\author{
Yelko Chento ${ }^{1, a}$, César Hueso ${ }^{1}$, Imanol Zamora ${ }^{2}$, Marco Fabbri², Cristina De La Fuente ${ }^{2}$ and Asier Larringan ${ }^{1}$ \\ ${ }^{1}$ IDOM Engineering, Consulting and Architecture S.A.U., Av. Zarandoa 2348015 Bilbao, Spain, +34 944797600 \\ 2IDOM Engineering, Consulting and Architecture S.A.U., Gran Vía Carlos III 9708028 , Barcelona, Spain, +34 934092222
}

\begin{abstract}
Jules Horowitz Reactor (JHR), a major infrastructure of European interest in the fission domain, will be built and operated in the framework of an international cooperation, including the development and qualification of materials and nuclear fuel used in nuclear industry. For this purpose UGXR Collimators, two multi slit gamma and Xray collimation mechatronic systems, will be installed at the JHR pool and at the Irradiated Components Storage pool. Expected amounts of radiation produced by the spent fuel and X-ray accelerator implies diverse aspects need to be verified to ensure adequate radiological zoning and personnel radiation protection. A computational methodology was devised to validate the Collimators design by means of coupling different engineering codes. In summary, several assessments were performed by means of MCNP5v1.60 to fulfil all the radiological requirements in Nominal scenario $(\mathrm{TEDE}<25 \mu \mathrm{Sv} / \mathrm{h})$ and in Maintenance scenario (TEDE $<2 \mathrm{mSv} / \mathrm{h}$ ) among others, detailing the methodology, hypotheses and assumptions employed.
\end{abstract}

\section{Introduction}

Jules Horowitz Reactor (JHR), built on the Cadarache site, will be a major infrastructure of European interest in the fission domain, open to the international collaboration. The JHR will be built and operated in the framework of an international cooperation between several organizations bound by a Consortium Agreement, one of whose main aim is the development and qualification of materials and nuclear fuel used in nuclear industry.

The necessities to design and build new material testing reactors to support operation of the existing power reactors fleets and qualification of future technologies systems have led to the development of precise mechatronic systems compliant not only with the experimental requirements but also with the very demanding nuclear safety constraints, such as UGXR Collimators. These two multi slit gamma and X-ray collimation systems, located at the JHR pool (RER) and Irradiated Components Storage pool (EPI) Fig-1 and Fig-2, will allow to carry out the collimation of the hard $\mathrm{X}$-rays towards the underwater part of the transmission imagining measurement system and the gamma beams between the immersed object to be measured and the experimental equipment located in the out of pile section, Fig-3.

These two Collimators, as well as support and hoisting structures for the gammametry detector, shall ensure proper shielding and shall also provide a second water barriers behind the pile liners.

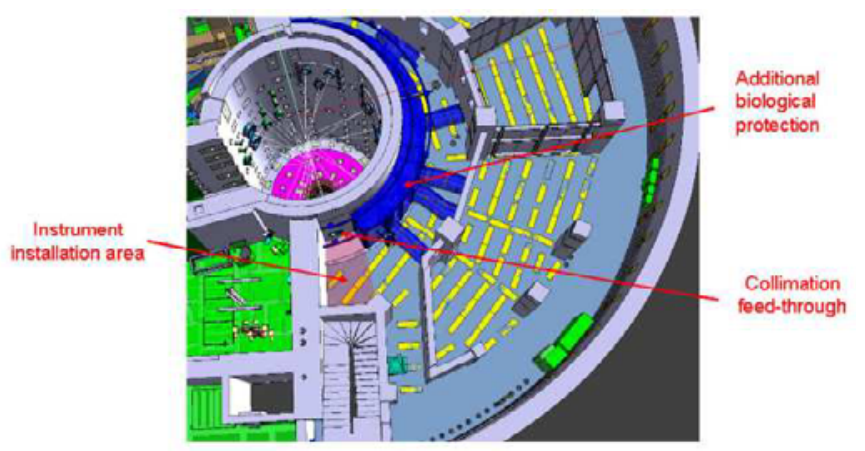

Figure 1. JHR pool Collimator.

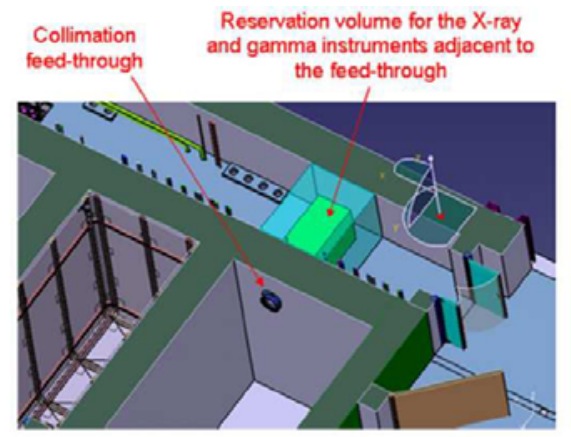

Figure 2. Irradiated Components Storage pool Collimator.

\footnotetext{
${ }^{\mathrm{a}}$ Corresponding author: yelko.chento@idom.com
} 
Several assessments are performed to fulfil all the radiological requirements, detailing the methodology employed as well as hypotheses and assumptions. Dose rate compliance is evaluated by mean of radiation transport calculations through MCNP5v1.60 [Ref. 1] and the use of SCALE6.1/ORIGEN-S [Ref. 2] for the source term definition, both intensity gamma and energy spectra.

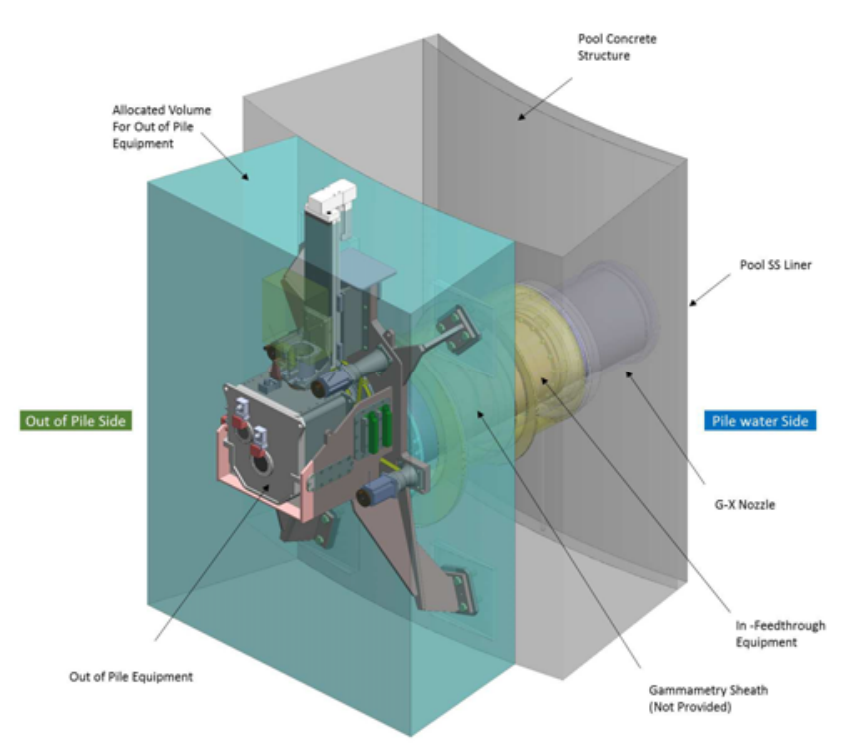

Figure 3. UGXR Collimators.

\section{Requirements}

Large amounts of radiation are expected to be produced by the spent fuel and X-ray accelerator, which implies that several aspects need to be verified to ensure adequate radiological zoning and radiation protection of the personnel. Three main requisites are defined that shall be taken into account during the design phase:

- A TEDE dose rate lower than $25 \mu \mathrm{Sv} / \mathrm{h}$ in the technical gallery concerning UGXR equipment in the out of pile section under normal conditions.

- Existence, under normal conditions, of a significant difference between the shielded flux and the collimated flux, considering the Lanthanum-140 energy peak as a reference value. As well, a non-significant scattered component in the flux detected.

- In Maintenance situation, a TEDE dose rate lower than $2 \mathrm{mSv} / \mathrm{h}$ in the area concerning UGXR equipment in the out of pile section under normal conditions.

The correct compliance of the model with the Verification requisites is ensured, during the design phase, taking into account three requirements groups:

- Safety analyses (Structural Integrity \& Biological Protection);

- Gamma viewing control (Geometrical viewing def. \& Absence of leakage path);

- Mechanical performance (Movements accuracy \& Drive dimensioning);

\section{Baseline data and hypotheses}

Multiple scenarios and hypotheses are defined that allowed to consider all the possible casuistry, so that even in the worst cases the Collimator adjacent galleries will be safe from the radiological point of view. The design shall ensure the dose rate requirements in:

- Nominal situation;

- Maintenance situation;

- Pre-collimator block loading/unloading situation;

- Other situations that shall be accomplished to satisfy the Nuclear Safety criteria (Leakage sensitivity analyses).

\section{Model definition}

The first step, avoiding the CAD generation by scratch, is to simplify and defeature UGXR Collimators models using SpaceClaim2015 [Ref. 3] package. The purpose of this is to allow successful conversion of the solid CAD bodies to MCNP5v1.60 [Ref. 1] through SuperMC [Ref. 4], obtaining a fast conversion process and also reducing the effort required to repair the resulting MCNP model.

It is convenient to remove details which are not important for radiation transport calculations, such as fillets, chamfers, bolt holes, etc. as shown in Fig-4. Whilst convertible to MCNP5v1.60 [Ref. 1], the presence of such details significantly increases calculation times.
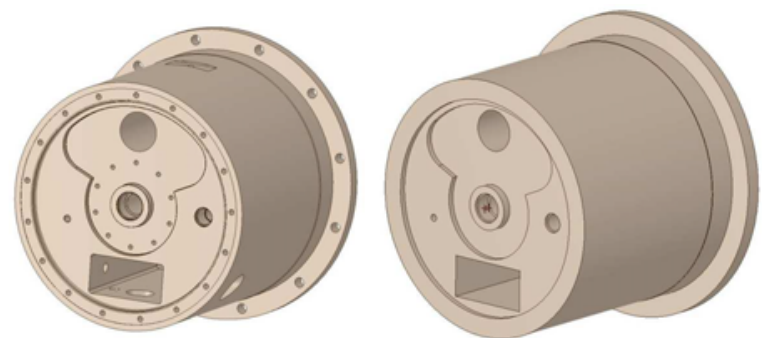

Figure 4. Comparison between original (left side) and simplified model.

The degree of simplification which is acceptable is a function of the photon importance of the features being considered. Generally, it is desirable to retain more detail of channels in the photon gradient direction, since photons would stream down them.

In order to maintain the correct mass of materials, such simplifications are always accompanied by homogenizations of materials and densities corrections. The geometry is modelled in a very detailed way, preserving the single components mass when possible.

Once the model is simplified and checked, the complex solids shall be decomposed into a combination of simple convex solids like box or sphere to allow the proper conversion by means of SuperMC [Ref. 4] to a Constructive Solid Geometry (CSG) required by MCNP5v1.60 [Ref. 1]. Therefore, manually assisted decomposition with SpaceClaim2015 [Ref. 3] is adopted 
and the whole model is decomposed into a number of convex solids which are then converted to MCNP files Fig-5.

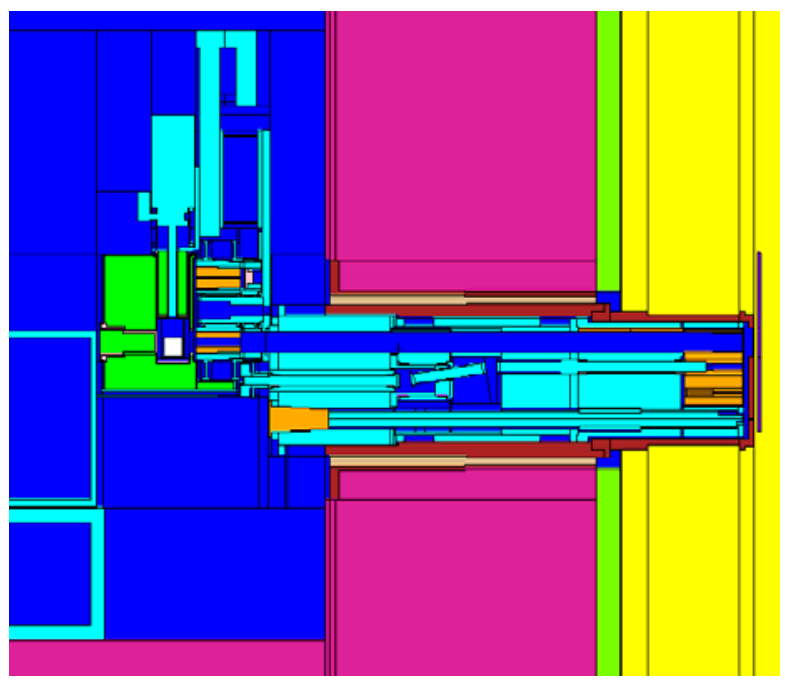

Figure 5. MCNP nominal model vertical cross section.

\section{Source term \& Radiation transport assessment}

\subsection{Source term}

The source term considered is based on the maximum fission product activities from an experimental PWR MOX fuel cooled for 3 days. The intensity of photons per rod emitted by the irradiated fuel is $1.605 \mathrm{E}+15$ photon $/ \mathrm{s}$ with an average energy around $0.150 \mathrm{MeV}$. The source term is modelled as a cylindrical fuel pin facing UGXR Collimators.

It has to be remarked that all the mentioned cases are assessed assuming the same conservative source term defined through the fuel activity concentration, determining the energy spectra by means of SCALE6.1/ORIGEN-S [Ref. 2].

\subsection{Radiation transport}

Radiation transport calculations, which are very computational demanding, are fundamental to accomplish with the dose rate zoning requirements. The radiation transport assessments, which allowed performing the dose rate evaluation to the JHR personnel, are carried out by means of MCNP5v1.60 [Ref. 1]. AP ICRP-119 TEDE conversion coefficient [Ref. 5] has been employed because they are the most conservative ones.

The detailed evaluation for each mentioned scenario is one of the keys of this methodology, so a previous CAD of each scenario of the preliminary Collimators design is required to elaborate the geometric model. An iterative procedure allowed the optimization of the design for each of the scenarios under study.
The MCNP5v1.60 [Ref. 1] model contains the UGXR Collimator, the technical gallery, the pool and its concrete wall, the fuel pin source, the $\mathrm{X}$-accelerator and its support. For conservatism no further objects are introduced in the technical gallery and the pin source is modelled without its support (see Fig-6). It has to be highlighted that from the radiological point of view, the internal equipment of the collimation feed-through plays an essential role in radiation protection in the out of pile section as it is the continuation of the biological protection formed by the concrete wall of the pool and thus it must ensure proper shielding.

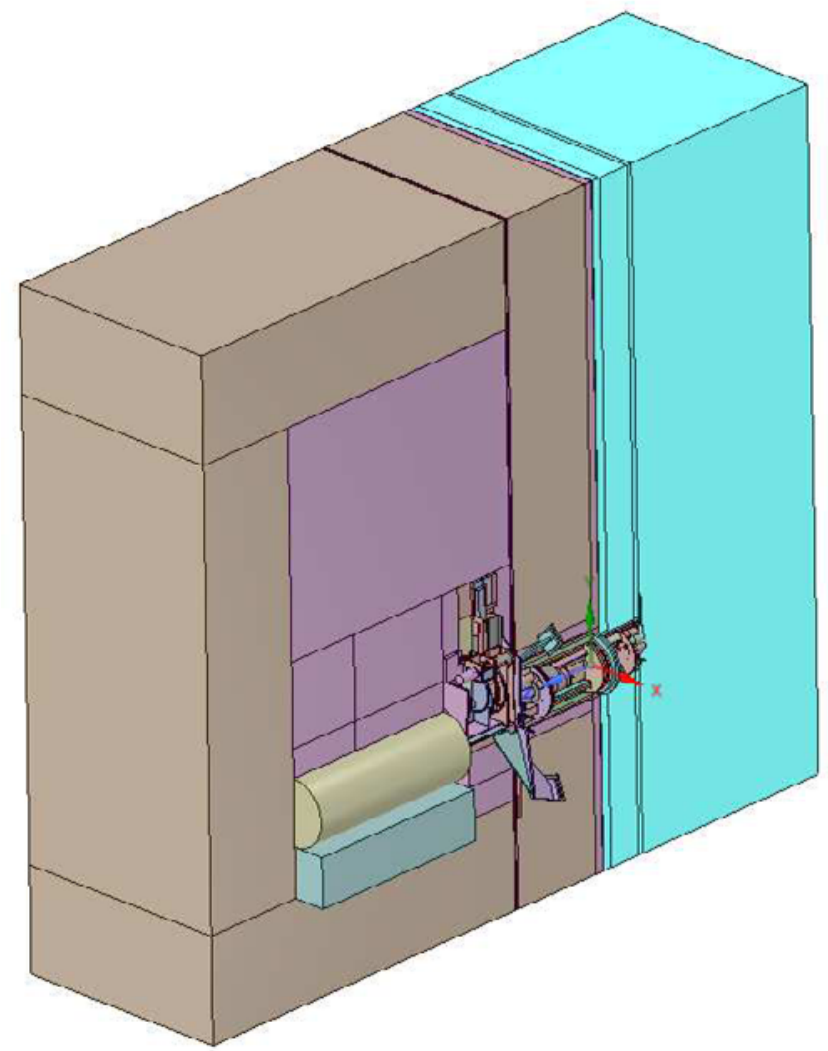

Figure 6. Slice view of the model.

Maintenance situation, Leakage situation and Precollimator block loading/unloading situation analyses introduce different changes with regarding Nominal situation model configuration, such as the modifications relative to the disposition of the fuel pin. These scenarios are complementary analyses to the Nominal situation where dose rate at technical gallery is calculated under different operational circumstances.

Considering different tallies outside and inside the Collimator allows to determine photon flux, thermal power and dose rate at different points of the Collimator but also in the technical gallery, where the JHR personnel is expected to be (see Fig-7). Furthermore, the radiological compliance has been assessed by the use of different MCNP5v1.60 [Ref. 1] tally typologies (e.g. F15/F25/F24) giving robustness to the analysis 


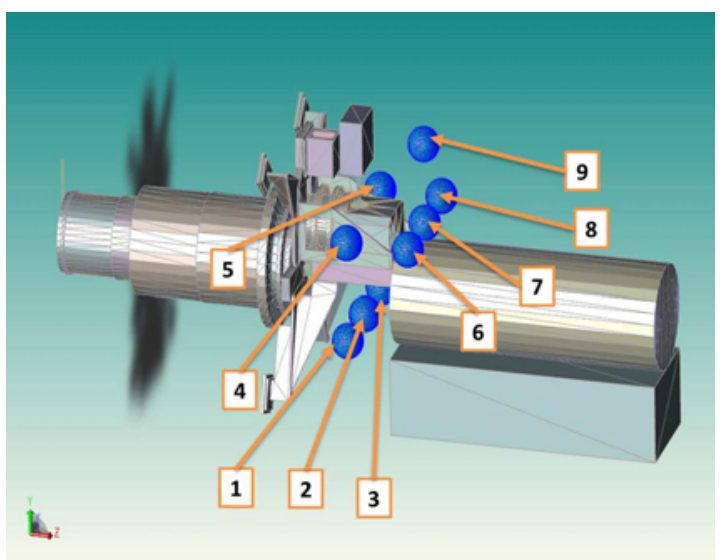

Figure 7. Some of the used tallies.

For the calculations, exponential transform variance reduction techniques are employed and optimized, as well as a mono-energetic weight window, by MCNP5v1.60 [Ref. 1] weight window generated previously, using a reduced density model. An iterative procedure allowed for the optimization of the variance reduction parameters, being lately checked via ADVANTG 3.0.3 code [Ref. 6] for each of the analyzed models.

\section{Results}

As expected, the dose rate decreases with the penetration inside the UGXR Collimators device due to the collimation effect and the shielding components. The primary photon dose contribution is found to be caused by the gamma direct coming from the source and they affect mostly the zone below the post-collimator wheel. Moreover, the secondary gamma in the technical gallery are generated almost all inside the lead detector shielding because of the large atomic number of the material.

For the "Nominal Situation" scenario, the dose rate estimated in the technical gallery is smaller than $25 \mu \mathrm{Sv} / \mathrm{h}$ required, except for a small not-accessible area. The higher dose rate values are located in the UGXR Collimators lower area due to a downside primary photon streaming caused by the scattering of the collimated gamma flux against the post-collimator wheel and collimator as shown in Fig-8, Fig-9, Fig-10 and Fig-11.

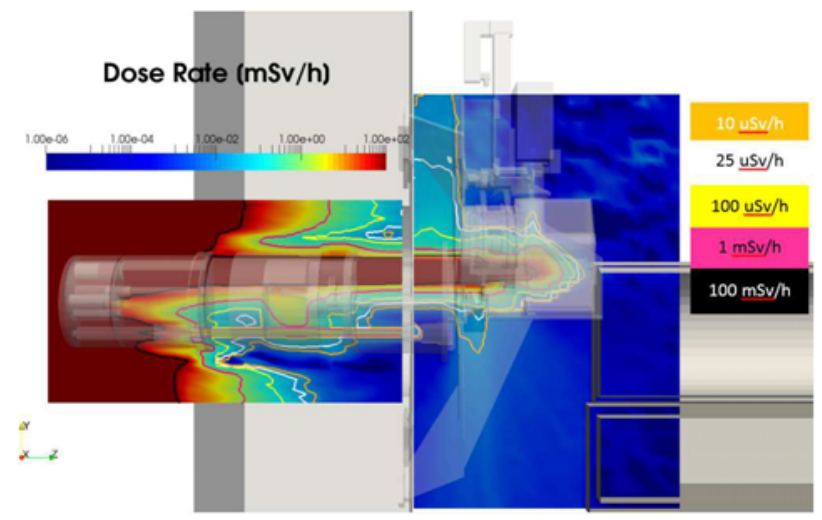

Figure 8. Dose Rate and contour plots for the Nominal situation.

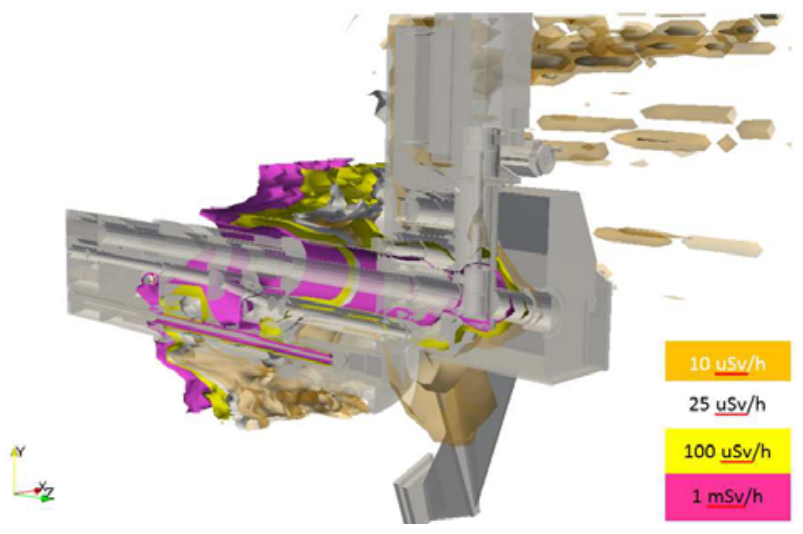

Figure 9. Dose Rate field - 3D contour plots for Nominal situation.

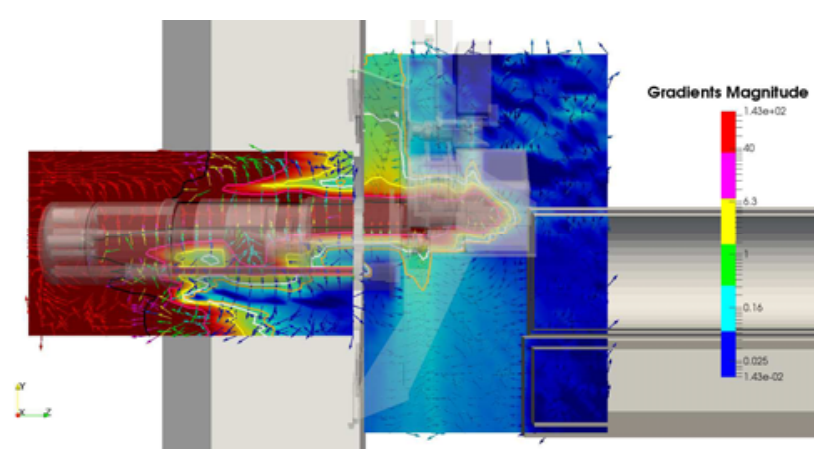

Figure 10. Dose Rate Gradient for Nominal situation.

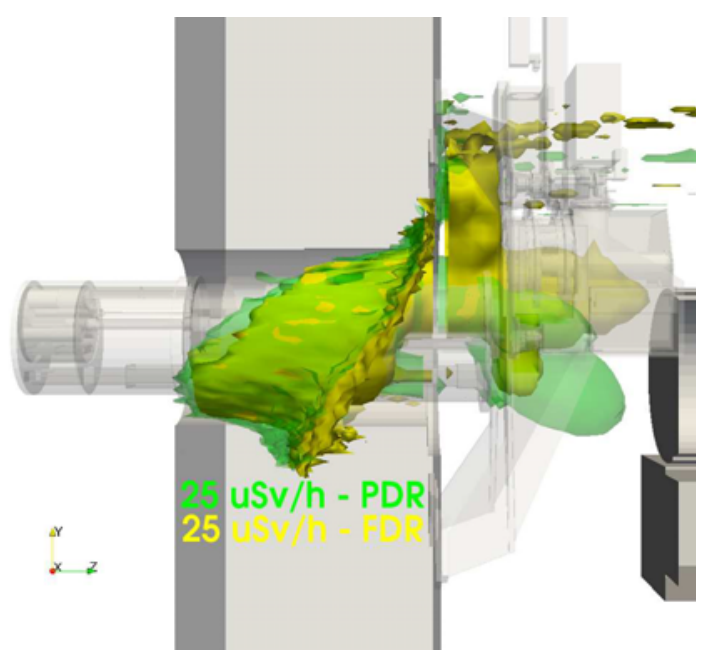

Figure 11. $25 \mu \mathrm{Sv} / \mathrm{h}$ Dose Rate 3D contour plot comparison between Final Design (FDR) and Preliminary Design (PDR) for Nominal situation.

Regarding the "Maintenance Situation", thanks to the innovative design solution proposed, the radiological zone classification could be declassified from yellow $(\mathrm{TEDE}<2 \mathrm{mSv} / \mathrm{h})$ to green (TEDE $<25 \mu \mathrm{Sv} / \mathrm{h})$ with a positive impact on the overall occupational dose for the personnel as shown in Fig-12 and Fig-13. It has to be remarked that only for the upper zone of the technical gallery a dose rate larger than $10 \mu \mathrm{Sv} / \mathrm{h}$ has been determined. 


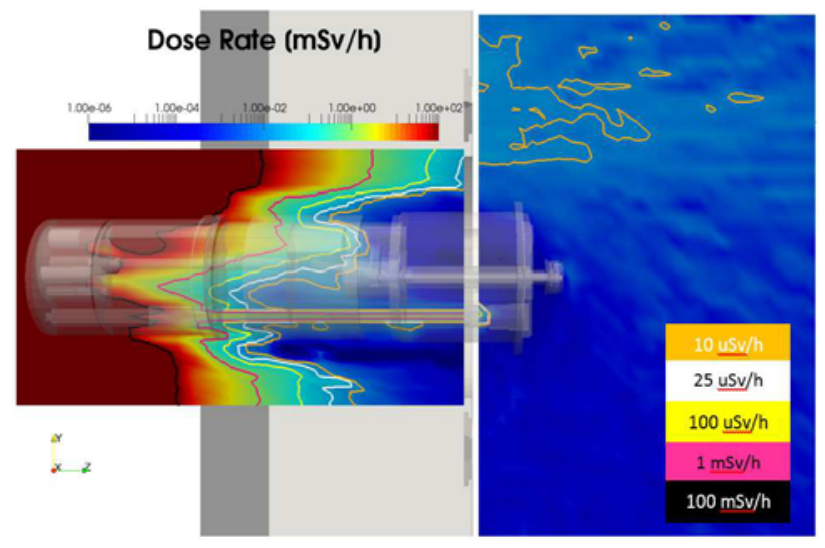

Figure 12. Dose Rate and contour plots for the Maintenance situation.

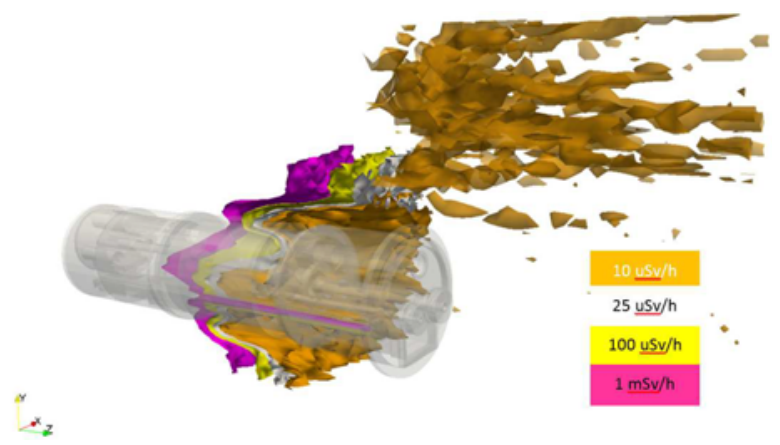

Figure 13. Dose Rate field - 3D contour plots for Maintenance situation.

From the leakage analyses it is noticeable the achieved design implicit safety shielding capacity of the UGXR Collimators and the concrete walls, not exceeding the $25 \mu \mathrm{Sv} / \mathrm{h}$ required, even for the maintenance case as shown in Fig-14. The main contribution of the dose rate in the technical gallery is due to the secondary photons generated by Bremsstrahlung, whereas the lower streaming, which is caused mostly due to the leakage of primary that photons is not relevant.

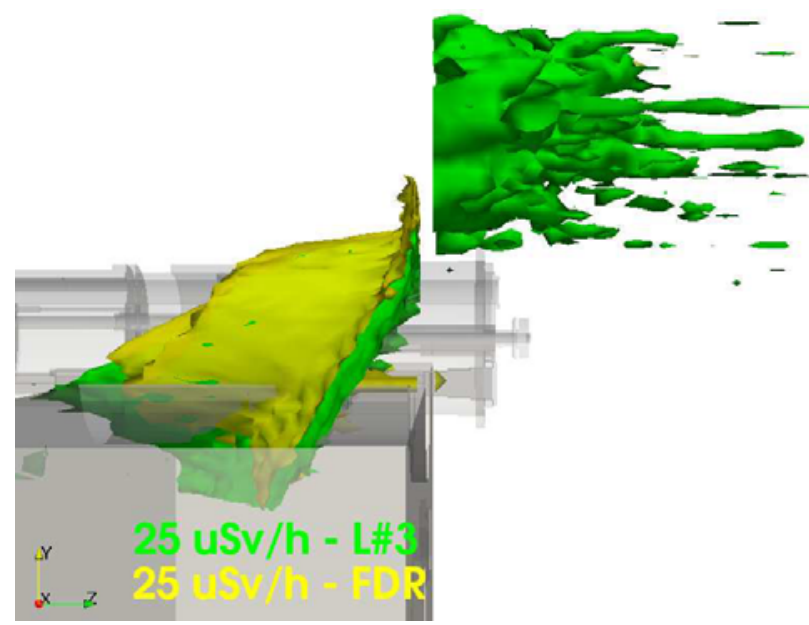

Figure 14. Dose Rate field - 3D contour plot comparison between Final Design (FDR) and Preliminary Design (PDR) for Leakage situation.
For the pre-collimator block loading/unloading operation, it has been confirmed the proper shielding capacity of the designed system as shown in Fig-15. The shielding action of both the pre-collimator utility head and the horizontal lead plug of the utility is evident, even when the precollimator is not present. Therefore, as expected, if the pre-collimator is present when the operation is started, the dose rates will be reduced by the extra-shielding.

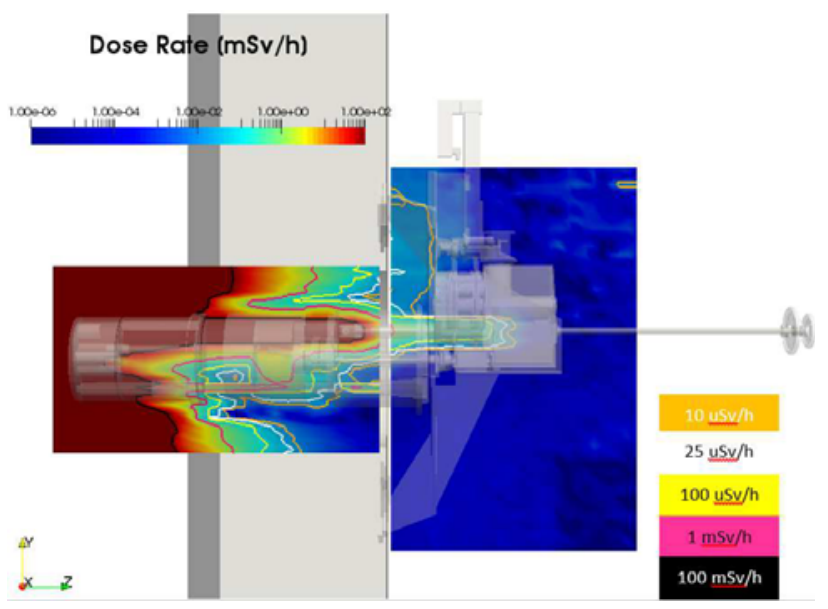

Figure 15. Dose Rate and contour plots for the Pre-collimator block loading/unloading situation.

Finally, a significant reduction of the photon flux of approximately 5 orders of magnitude is observed between the shielded flux and the collimated flux, in addition to a low scattered photon flux. Thus the collimation capacity of the UGXR Collimators is assured.

\section{Conclusions}

UGXR Collimators, located at the JHR pool and Irradiated Components Storage pool, dose rate compliance calculations are assessed by means of different engineering computational codes. The model Verification requirements has been satisfied, being able to verify the radiological design of the UGXR Collimators. Calculation codes selected for the development of this methodology are widely available, internationally used and validated in many studies.

It can be concluded that the UGXR Collimators design fulfils the dose requirement both for "Nominal and Maintenance Situation" including when applying conservatisms, as well as for the rest of the scenarios taken into consideration. Furthermore, the collimation capacity of the UGXR Collimators is checked through the significant difference between shielded and collimated flux, resulting in a flux incident to the crystal detector with a low scattered component and therefore less noise to signal will be observed.

It is worth to point out that several iterations are required until the design of the UGXR Collimators dose rate restrictions and optimal collimation capacity have been accomplished. 


\section{References}

1. Los Alamos National Laboratory. MCNP - A General N-Particle Transport Code, Version 5 Volume I: Overview and Theory, X-5 Monte Carlo Team, LA-UR-03-1987, April 2003.

2. Oak Ridge National Laboratory, SCALE: A Comprehensive Modeling and Simulation Suite for Nuclear Safety Analysis and Design, June 2011.

3. SpaceClaim Corporation, SpaceClaim 2015.

4. FDS, SuperMC (Monte Carlo Automatic Modeling Program for Radiation) 5.1 Professional, 2015.

5. ICRP, International Commission on Radiological Protection. ICRP-119, Compendium of Dose Coefficients based on ICRP Publication 60.

6. Oak Ridge National Laboratory, ADVANTG 3.03 Automated Variance Reduction Generator. 